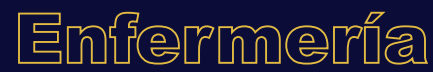

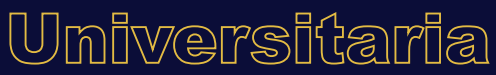

\section{Persistencia microbiana de importancia intrahospitalaria en uniformes clínicos a raíz de la pandemia de COVID-19}

\author{
Microbial persistence in clinical uniforms: \\ consideration of the COVID-19 pandemic
}

Persistência microbiana de importância intra-hospitalar em uniformes clínicos como resultado da pandemia da COVID-19

\section{S. Kappes}

ORCID

${ }^{\mathrm{a}}$ 0000-0001-8101-3898

Universidad San Sebastian, Facultad de Ciencias para el cuidado de la Salud, Chile

Recibido: 22 septiembre 2020

Aceptado: 01 mayo 2021

\section{RESUMEN}

Introducción: El lavado de manos es la medida que más impacta en la prevención de infecciones asociadas a la atención en salud. Hay poca evidencia del rol que cumplen los uniformes clínicos como vector en transmisión cruzada de infecciones en hospitales.

Método: Se realizó una revisión rápida con criterios Cochrane y lista de chequeo PRISMA con acceso a bases de datos PubMed, Ovid, ProQuest y Google Académico en español e inglés del 2010-2020. El objetivo establecido fue buscar evidencia sobre la persistencia de microorganismos de importancia intrahospitalaria en uniformes clínicos, a raíz de la actual pandemia de COVID-19. El análisis crítico de los artículos se realizó con ayuda de herramientas del Joanna Briggs Institute (checklist estudios analíticos, serie de casos, texto y opinión, ensayos clínicos controlados y revisiones sistemáticas). 
Resultados: En la búsqueda inicial se obtuvieron 1703 artículos, de los cuales fueron seleccionados 8. Se encuentra evidencia de presencia de microorganismos en uniformes clínicos. Las zonas más contaminadas son bolsillos y mangas. MERS-COV y SARS-COV han mostrado persistencia en aluminio hasta 48 horas, madera 4 días, papel hasta 24 horas. En género, el SARS-COV-2 ha demostrado una persistencia de 2 días.

Conclusiones: Existe evidencia de la persistencia de microrganismos bacterianos y virales en uniformes clínicos. Los lugares más contaminados son bolsillos y mangas. Al contacto está demostrada la presencia de microrganismos en uniformes clínicos horas después. Se deben implementar medidas que tomen en cuenta el rol potencial de transmisión de patógenos durante el uso de uniformes clínicos en hospitales.

Palabras claves: Infección hospitalaria; vestuario; transmisión de enfermedad infecciosa; infecciones por coronavirus; personal de enfermería en hospital; Chile.

\section{ABSTRACT}

Introduction: Hand washing is the measure that has the greatest impact on the prevention of infections associated with health care. There is little evidence of the role of clinical uniforms as a vector in cross-transmission of infections in hospitals.

Method: A rapid review was carried out with Cochrane criteria and PRISMA check list with access to Pubmed, Ovid, ProQuest and Google Academic databases in Spanish and English from 2010-2020. The objective of the review was to search for evidence of the persistence of microorganisms of intra-hospital importance in clinical uniforms, in light of the current Covid-19 pandemic. The critical analysis of the articles was carried out with tools from the Joanna Briggs Institute (checklist analytical studies, case series, text and opinion, controlled clinical trials and systematic reviews)

Results: The initial search yielded 1703 articles, of which 8 articles were selected. Evidence of persistence of microorganisms is found in clinical uniforms. The most contaminated areas are pockets and sleeves. MERS-COV and SARS-COV, have shown persistence in aluminum up to 48 hours, wood 4 days, paper 24 hours. In genus, SARS-COV 2 has shown a persistence of 2 days.

Conclusions: There is evidence of persistence of bacterial and viral microorganisms in clinical uniforms. The most contaminated places are pockets and sleeves. After the position, hours later there is evidence of the presence of microorganisms in clinical uniforms.

Keywords: Cross infection; clothing; disease transmission, infectious; coronavirus infections; nursing staff, hospital; Chile.

\section{RESUMO}

Introdução: A lavagem das mãos é a medida de maior impacto na prevenção de infecções associadas aos cuidados de saúde. Existem poucas evidências sobre o papel que desempenham os uniformes clínicos como vetor na transmissão cruzada de infecções em hospitais. Método: Foi realizada uma revisão rápida com os critérios Cochrane e checklist PRISMA com acesso às bases de dados PubMed, Ovid, ProQuest e Google Académico em espanhol e inglês de 2010-2020. O objetivo estabelecido foi buscar evidência sobre a persistência de microrganismos de importância intra-hospitalar em uniformes clínicos, como resultado da atual pandemia de COVID-19. A análise crítica dos artigos foi realizada com ferramenta do Joanna 
Briggs Institute (checklist de estudos analíticos, série de casos, texto e opinião, ensaios clínicos controlados e revisões sistemáticas).

Resultados: A busca inicial resultou em 1703 artigos, dos quais foram selecionados 8. Foram encontradas evidências de persistência de microrganismos em uniformes clínicos. As áreas mais contaminadas são os bolsos e as mangas. MERS-COV e SARS-COV mostraram persistência em alumínio até 48 horas, madeira 4 dias, papel até 24 horas. No gênero, o SARS-COV-2 mostrou uma persistência de 2 dias.

Conclusões: Existe evidência da persistência de microrganismos bacterianos e virais em uniformes clínicos. Os locais mais contaminados são os bolsos e as mangas. Uma vez colocado, é demonstrada a presença de microrganismos em uniformes clínicos horas depois. Devem ser implementadas medidas que tomem em conta o papel potencial de transmissão de patógenos de uniformes clínicos em hospitais.

Palavras-chave: Infecção hospitalar; vestuário; transmissão de doença infecciosa,; infecções por coronavírus; recursos humanos de enfermagem no hospital, Chile.

\section{INTRODUCCIÓN}

Las infecciones asociadas a la atención en salud (IAAS) son un problema global y han sido reportadas como un evento adverso frecuente en los hospitales del mundo ${ }^{1,2}$. Un estudio reciente realizado en 137 países demuestra un índice mayor de muertes por malos servicios sanitarios, que por no tener acceso a ellos3. Las IAAS generan costos directos que hacen más caros los sistemas de salud y determinan mayor morbimortalidad para los pacientes 4.5 .

En el contexto actual en medio de la pandemia por COVID-19, los hospitales han reportado una ocupación completa ${ }^{6}$, incluso a veces mayor a su capacidad en servicios de alta complejidad, unidades de cuidados intensivos y servicios de urgencia7. Sumado al agotamiento del personal médico y de enfermería, también se ha producido en los pacientes con COVID-19 infecciones por otros patógenos endémicos de los hospitales como Pseudomona aeruginosa y Escherichia coli ${ }^{8}$. Esta situación es grave ya que las infecciones hospitalarias en pacientes con COVID-19 aumentan aún más su gravedad, estadía hospitalaria, consumo de medicamentos y mortalidad9.

El lavado de manos está ampliamente estudiado como la medida con mayor impacto en la prevención de las infecciones asociadas a la atención en salud ${ }^{10}$. Poca evidencia hay acerca del rol que cumple la vestimenta médica y de enfermería como vector para la transmisión cruzada de infecciones en hospitales ${ }^{11}$. En este sentido, es importante determinar la persistencia de microorganismos en delantales y uniformes con el fin de orientar a mejorar el diseño de estrategias para su uso en hospitales, tiempo necesario de recambio, telas adecuadas para disminuir la adherencia de microorganismos y otras medidas.

Dentro de los hospitales se ha normado el uso de elementos de protección personal (EPP) ante el contacto con pacientes diagnosticados o sospechosos de portar SARS-COV-2. Sin embargo, estudios han mostrado que el mayor problema de contaminación para el personal médico se produce al colocar o retirar los EPP, con el riesgo de contaminar las manos o el uniforme del operador y la potencial transmisión tanto al personal como a otros pacientes ${ }^{12}$. Además, muchos hospitales se han visto enfrentados a la falta de EPP y a restringir su uso, únicamente disponibles para el personal de las Unidades de Cuidado Intensivo o laboratorios de procesamiento de muestras ${ }^{13}$. 
Todo lo relatado muestra la relevancia de conocer la persistencia de virus y bacterias en uniformes o delantales ya que pueden jugar un rol importante en las infecciones intrahospitalarias. Por consiguiente, el objetivo de esta investigación es identificar evidencia disponible acerca de la persistencia microbiana de importancia intrahospitalaria en uniformes clínicos y delantales, a la luz de la actual pandemia de COVID-19.

\section{METODOLOGÍA}

Se realizó una revisión de alcance (scope review). Para esta propuesta metodológica se estableció la siguiente pregunta de investigación: ¿existe evidencia de la persistencia de microorganismos de importancia intrahospitalaria en delantales y uniformes clínicos en la actual pandemia de COVID-19?

La estrategia de búsqueda fue específica para una revisión rápida con los criterios que establece la colaboración Cochrane ${ }^{14}$ y la lista de chequeo PRISMA ${ }^{15}$. La búsqueda incluyó las bases de datos PubMed, Ovid, ProQuest y Google Académico, en idiomas español e inglés, periodo de búsqueda en años 2010-2020. Las palabras claves para la búsqueda fueron seleccionadas de los descriptores en ciencias de la salud: infección hospitalaria, infecciones por coronavirus, vestuario. Se utilizaron los operadores booleanos $[A N D]$ y $[O R]$ con los criterios infección hospitalaria $[A N D]$ vestuario $[O R]$ uniformes $[A N D]$ infecciones por coronavirus.

Criterios de inclusión: Se incluyen todo tipo de artículos científicos (independientemente de su diseño), que den cuenta de investigación en seres humanos y evidencien en sus resultados persistencia de microorganismos, bacterianos o virales, en delantales clínicos o uniformes. Los criterios de exclusión comprenden: comentarios o respuestas a editoriales, artículos duplicados.

Para delimitar la inclusión de los artículos se siguieron los criterios de calidad del Joanna Briggs Institute ${ }^{16}$. Se realizó una evaluación inicial estableciendo el criterio en cada checklist del cumplimiento del 60\% de los ítems para considerar que el artículo es de buena calidad, en todo momento se siguió la recomendación de Chan et al. ${ }^{17}$. Posteriormente, una segunda evaluación fue hecha por otro revisor y se definió acuerdo en aquellos checklist en los cuales se presentaron diferencias entre ambos evaluadores en cuanto al cumplimiento de las listas de chequeo.

\section{RESULTADOS}

El concepto central a analizar en los resultados fue la evidencia acerca de la persistencia de microorganismos bacterianos y virales en uniformes, así como las posibles diferencias según la tela, zonas más contaminadas, entre otros.

La búsqueda inicial entregó 1703 artículos. Para Google Académico 442 artículos, 19 artículos en PubMed, 1232 en ProQuest, 10 en Ovid. Luego de excluir comentarios de editoriales, duplicados y artículos que no estaban en el objetivo de la investigación, se analizaron 8 artículos como se muestra en la figura 1.

Para el análisis de las evidencias se procedió a la lectura del artículo completo, donde se extraen los principales hallazgos que se presentan en la tabla 1. De los artículos incluidos hay 5 revisiones (narrativas, sistemáticas), un estudio descriptivo, un ensayo clínico controlado y un estudio correlacional. En los artículos seleccionados, 6 de ellos reportan la persistencia de bacterias de importancia nosocomial en delantales y sólo 2 sobre microorganismos virales. 


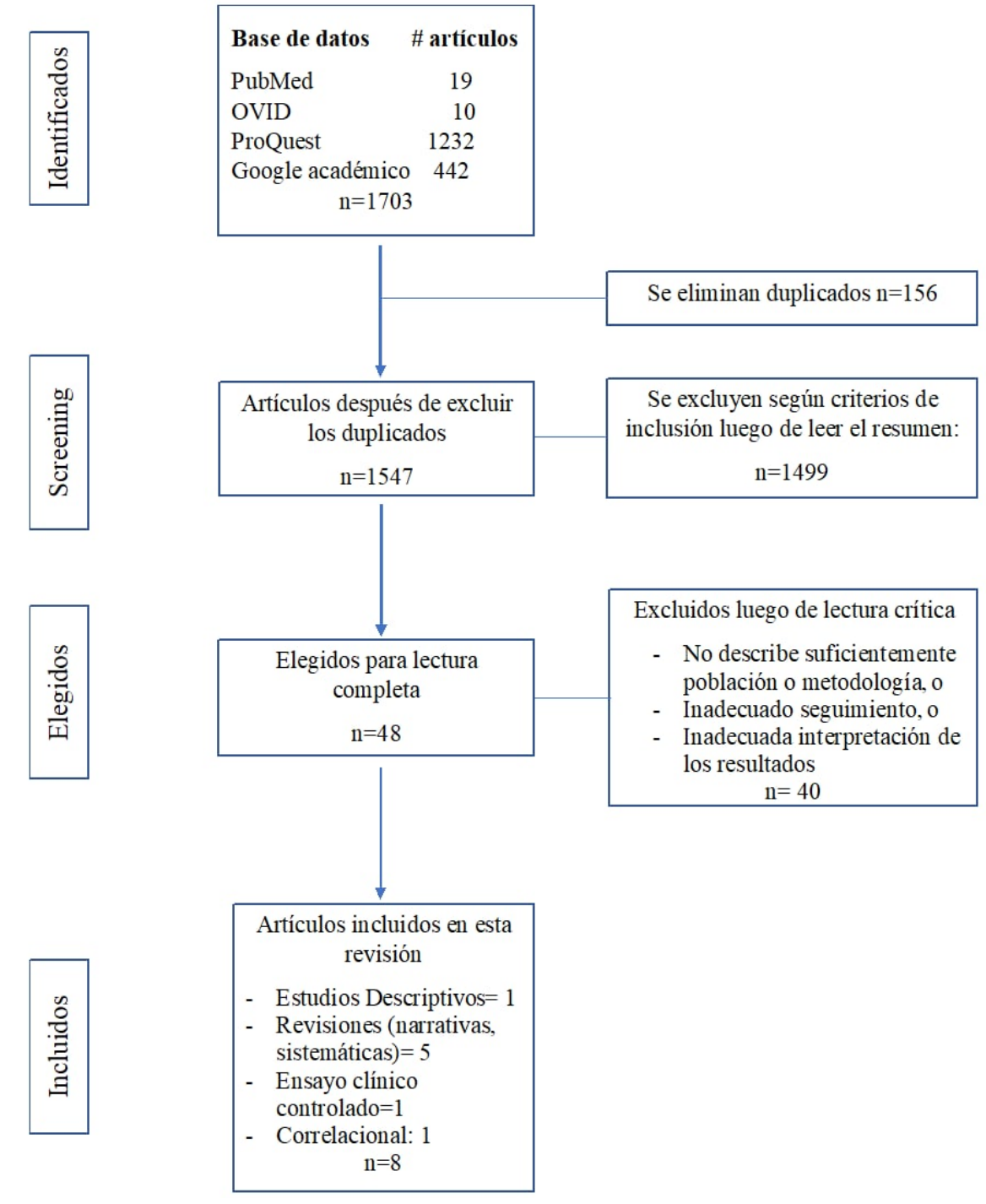

Figura 1. Flujograma selección de artículos PRISMA

\section{Persistencia de bacterias en uniformes clinicos}

Existe evidencia de que los lugares más contaminados son las mangas y bolsillos ${ }^{18}$, aunque se encuentren recién lavados, solo en un par de horas es posible recuperar bacterias como Acinetobacter baumannii y Pseudomona aeruginosa ${ }^{19}$. No hay diferencia con respecto a la tela con la que está fabricado el uniforme clínico en relación con la persistencia de microorganismos ${ }^{20}$.

La literatura también da cuenta de otras bacterias que han podido ser recuperadas de delantales y uniformes clínicos como Escherichia coli, Enterobacter spp. y Staphylococcus aureus ${ }^{20}$. La revisión sistemática de Haun ${ }^{21}$ muestra que la bacteria más comúnmente aislada en uniformes es Staphylococcus aureus. Dicho hallazgo es importante ya que esta bacteria (especialmente la cepa resistente a la Vancomicina) es responsable de muchas infecciones intrahospitalarias, sobre todo en los pacientes más susceptibles²2. 
Tabla 1. Estudios incluidos y sus principales hallazgos

\begin{tabular}{|c|c|c|c|}
\hline Autores & $\begin{array}{c}\text { Año de } \\
\text { publicación }\end{array}$ & $\begin{array}{l}\text { Diseño } \\
\text { del estudio }\end{array}$ & Principales hallazgos \\
\hline Bearman, et al. ${ }^{18}$ & 2014 & $\begin{array}{l}\text { Revisión } \\
\text { narrativa }\end{array}$ & $\begin{array}{l}\text { La frecuencia de aparición de microorganismos } \\
\text { multirresistentes está inversamente relacionada } \\
\text { con mayor frecuencia de cambio y de lavado. Mayor } \\
\text { contaminación en mangas. }\end{array}$ \\
\hline Munoz-Price, et al. ${ }^{19}$ & 2012 & $\begin{array}{l}\text { Estudio } \\
\text { correlacional }\end{array}$ & $\begin{array}{l}\text { La presencia de Acinetobacter en las manos del } \\
\text { personal se asoció con mayor probabilidad a la } \\
\text { presencia de Acinetobacter en los delantales } \\
(p<0.001) \text {. }\end{array}$ \\
\hline Goyal, et al. ${ }^{20}$ & 2019 & $\begin{array}{l}\text { Revisión } \\
\text { sistemática }\end{array}$ & $\begin{array}{l}\text { De los } 11 \text { estudios que identifican microorganismos } \\
\text { en delantales, } 8 \text { presentan cultivos positivos para } E \text {. } \\
\text { aureus, } 3 \text { para Acinetobacter, } 6 \text { para Pseudomona y } \\
5 \text { estudios para Enterobacter spp. No hay diferencia } \\
\text { concluyente respecto a que la tela de los delantales/ } \\
\text { uniformes clínicos (algodón, poliéster, o mezcla) } \\
\text { presente diferencias en relación con los cultivos. }\end{array}$ \\
\hline Haun, et al. ${ }^{21}$ & 2016 & $\begin{array}{l}\text { Revisión } \\
\text { sistemática }\end{array}$ & $\begin{array}{l}\text { Por técnica de hisopado simple el microorganismo } \\
\text { más común detectado en los uniformes clínicos y } \\
\text { delantales fue } E \text {. aureus. }\end{array}$ \\
\hline Kampf, et al. ${ }^{23}$ & 2020 & $\begin{array}{l}\text { Revisión } \\
\text { narrativa }\end{array}$ & $\begin{array}{l}\text { Virus MERS-COV y SARS-COV han mostrado } \\
\text { persistencia en aluminio hasta } 48 \text { horas, madera hasta } \\
4 \text { días, papel hasta } 24 \text { horas. }\end{array}$ \\
\hline Aboubakr, et al. ${ }^{24}$ & 2020 & $\begin{array}{l}\text { Revisión } \\
\text { narrativa }\end{array}$ & $\begin{array}{l}\text { El virus SARS COV-2 ha demostrado mayor } \\
\text { supervivencia en superficies porosas que en las no } \\
\text { porosas. Persistencia hasta } 2 \text { días en ropa. }\end{array}$ \\
\hline Wiener-Well, et al. ${ }^{25}$ & 2011 & $\begin{array}{l}\text { Estudio } \\
\text { descriptivo }\end{array}$ & $\begin{array}{l}58 \% \text { del personal afirma cambiar su uniforme en } \\
\text { forma diaria. En } 63 \% \text { de los delantales se aislaron } \\
\text { bacterias potencialmente patógenas. }\end{array}$ \\
\hline Burden, et al. ${ }^{29}$ & 2011 & $\begin{array}{l}\text { Ensayo clínico } \\
\text { controlado }\end{array}$ & $\begin{array}{l}\text { Uniformes recién lavados tienen } 0 \text { colonias de } \\
\text { microorganismos. A las } 3 \text { horas ya tienen } 50 \% \text { del } \\
\text { recuento de colonias que tendrá a las } 8 \text { horas de uso. }\end{array}$ \\
\hline
\end{tabular}

\section{Persistencia de virus en uniformes y delantales}

En cuanto a los virus, se ha demostrado que partículas virales pueden ser recuperadas de diferentes superficies como aluminio hasta 48 horas, madera inclusive 4 días, papel por 24 horas ${ }^{23}$. Esto es válido para los virus SARS-COV y MERS-COV, de los cuales se tenía información previa a la pandemia por SARS-COV-2 que produce la enfermedad COVID-19. El virus SARS-COV-2 ha demostrado mayor supervivencia en superficies porosas que en las no porosas, con una persistencia de hasta 2 días en ropa ${ }^{24}$.

\section{DISCUSIÓN}

A pesar de que la persistencia de microorganismos en diferentes superficies ha sido documentada, no hay suficientes estudios que reporten la presencia en delantales o uniformes clínicos. Este déficit es mayor cuando se refiere a agentes virales.

Otro punto importante es la asociación entre contaminación de las manos de los trabajadores de salud con la de los uniformes y delantales. Un estudio realizado en EE. UU. determinó la presencia 
de Acinetobacter baumannii en las manos de los trabajadores de salud y los delantales, no así para los uniformes clínicos ${ }^{19}$.

Una investigación realizada en Israel muestra la carga bacteriana presente en uniformes del personal médicos y enfermería, obtenidos mediante cultivo simple. Los hallazgos determinaron que en $63 \%$ de los uniformes se aisló al menos una bacteria potencialmente patógena, a pesar de que $58 \%$ de los participantes declararon cambiar su uniforme diariamente ${ }^{25}$. Destaca el crecimiento de Acinetobacter baumannii, Staphylococcus aureus, Enterobacter spp y Pseudomona aeruginosa, patógenos que han sido relacionados con infecciones asociadas a la atención en salud 26-28.

En un estudio llevado a cabo en Reino Unido determinó que la contaminación de delantales y uniformes con Staphylococcus aureus va del 5 al 29\%. El estudio asocia mayor contaminación de delantales y uniformes con mayor uso, trabajo directo con pacientes, y ciertas áreas de mayor contaminación como las mangas. Además, la aparición de microorganismos multirresistentes está inversamente relacionada con mayor frecuencia de cambio y de lavado ${ }^{18}$. Dentro de esta revisión se determina que un uniforme limpio también se contamina tras su uso por algunas horas, lo que reafirma su importancia como posible vector ${ }^{29}$.

Una importante revisión sistemática que incluyó estudios desde 1990-2018 identificó 214 reportes con el tema principal, la contaminación microbiológica de delantales y uniformes; 33 fueron revisiones sistemáticas. De los 11 estudios que identifican microorganismos en delantales, 8 presentan cultivos positivos para Staphylococcus aureus, 3 para Acinetobacter baumannii, 6 para Pseudomona aeruginosa y para Enterobacter spp 5 estudios ${ }^{20}$.

Asimismo, se han desarrollado investigaciones similares en EE. UU, Asia, Europa, África, Australia y muy pocos en Sudamérica. En los estudios antes mencionados las técnicas más comunes para determinar la presencia de microorganismos en los delantales y uniformes fue tomando muestras mediante hisopado de diferentes zonas (mangas, bolsillos, zona abdominal) o colocando directamente el espécimen sobre una placa de cultivo, comúnmente agar sangre o con la adición de algún medio según el patógeno de interés ${ }^{21}$.

Estudios de virus con MERS-COV y SARS-COV han mostrado persistencia en aluminio por 48 horas, madera hasta 4 días, papel hasta 24 horas, bajo ciertas condiciones de temperatura. Así, se ha probado que estos virus pueden persistir hasta 9 días en superficies a temperatura ambiente ${ }^{21}$.

En relación con SARS-COV-2 no conocemos su persistencia en delantales y uniformes clínicos. Sin embargo, varios estudios apuntan a que los trabajadores de salud podemos facilitar la transmisión de este patógeno. La persistencia de SARS-COV-2 en textiles ha sido documentada hasta por 2 días, aunque pueden existir diferencias según la humedad y temperatura²3.

Hasta antes de la aparición de la pandemia por COVID-19, en algunos países de Latinoamérica era común ver a los trabajadores de salud fuera de los hospitales con uniformes clínicos. Sin embargo, la facilidad de transmisión del virus y las recomendaciones de la $\mathrm{OMS}^{30}$ acerca de las precauciones que deben tener los trabajadores de salud ha marcado un cambio de conducta. Por ello, las recomendaciones actuales deben incluir el manejo adecuado de los con uniformes clínicos y delantales en los cuales puede persistir el virus SARS-COV-2. Los uniformes usados deben ser transportados en bolsas y hacer énfasis en el lavado de manos luego de su manipulación. Para su tratamiento es suficiente el lavado en lavadora con detergente y ciclo habitual de 60-90 minutos ${ }^{30}$.

Ahora bien, las limitaciones del presente trabajo están dadas por la heterogeneidad de los estudios, lo que no permite hacer un análisis mayor. Por consiguiente, es necesaria más investigación en torno a la persistencia de los virus en diferentes superficies, la cual se podría llevar a cabo con 
técnicas de metagenómica. Esta técnica analiza mediante secuenciación masiva de ADN de todos los genomas presentes en una muestra, con lo cual se podrá identificar especies no habituales de bacterias y virus ${ }^{31,32}$.

Es probable que se abra la necesidad de uso de uniformes clínicos o delantales con nanopartículas de metales, las cuales han demostrado ser eficientes en disminuir la adherencia de microorganismos a sus fibras. Estos tejidos funcionales ya están siendo utilizados en la fabricación de ropa de cama para hospitales $33-35$.

Quedan aún años de investigación, muy necesarios ya que una vez superada esta pandemia podrá seguir otra, con nuevos desafíos para la ciencia médica y de enfermería, pero con lecciones que podemos aprender desde ahora.

\section{CONCLUSIONES}

Existe evidencia de la persistencia de varios microorganismos bacterianos y virales en los delantales clínicos y uniformes del personal, siendo las zonas más contaminadas tanto mangas como bolsillos. La contaminación de ellos con microorganismos ocurre sólo horas después de puestos, por lo que deben considerarse un potencial vector. Se deben implementar medidas en los programas de control de infecciones que tomen en cuenta el rol potencial de transmisión de patógenos a contar de delantales y uniformes en hospitales.

\section{RESPONSABILIDADES ÉTICAS}

Protección de personas y animales. Los autores declaran que en este estudio no se realizaron experimentos con humanos o animales.

Confidencialidad. Los autores declaran que el estudio contiene datos sensibles.

Conflicto de intereses. Los autores declaran que no tienen conflictos de intereses.

Financiamiento. Los autores declaran que este estudio no requirió de financiamiento.

\section{REFERENCIAS}

1. Vergara T, Fica A. Estudio de costo de las infecciones del torrente sanguíneo asociadas a catéter vascular central en pacientes adultos en Chile. Rev. chil. infectol. 2015; 32(6): 634-8.

http://dx.doi.org/10.4067/SO716-10182015000700004

2. Saavedra $\mathrm{CH}$, Ordóñez KM, Díaz JA. Impacto de la infección nosocomial en un hospital de Bogotá (Colombia): efectos en mortalidad y costos. Rev. chil. infectol. 2015; 32(1): 25-9.

http://dx.doi.org/10.4067/SO716-10182015000200004

3. Kruk ME, Gage AD, Joseph NT, Danaei G, García-Saizó S, Salomon JA. Mortality due to low-quality health systems in the universal health coverage era: A systematic analysis of amenable deaths in 137 countries. Lancet. 2018; 392(10160): 2203-12.

https://doi.org/10.1016/S0140-6736(18)31668-4

4. Callejas-Díaz A, Fernández-Pérez C, Ramos-Martínez A, Múñez-Rubio E, Sánchez-Romero I, Vargas -Núñez JA. Impacto de la bacteriemia por Pseudomonas aeruginosa en un hospital de tercer nivel: mortalidad y factores pronósticos. Med. clín. 2019; 152(3): 83-9.

https://doi.org/10.1016/j.medcli.2018.04.020

5. Hollenbeak CS, Schilling AL. The attributable cost of catheter-associated urinary tract infections in the United States: A systematic review. Am J Infect Control. 2018; 46(7): 751-7.

https://doi.org/10.1016/j.ajic.2018.01.015 
6. Peña VH, Espinosa A. Modelamiento predictivo para el cálculo de demanda de camas hospitalarias de cuidados intensivos a nivel nacional en el marco de la pandemia por COVID-19. Medwave. 2020; 20(9). https://doi.org/10.5867/medwave.2020.09.8039

7. Oliveira-da Costa Lino D, Barreto R, de Souza FD, Mota-de Lima CJ, Bezerra-da Silva Junior G. Impact of lockdown on bed occupancy rate in a referral hospital during the COVID-19 pandemic in northeast Brazil. Braz J Infect Dis. 2020; 24(5): 466-9. https://doi.org/10.1016/j.bjid.2020.08.002

8. Garcia-Vidal C, Sanjuan G, Moreno-García E, Puerta-Alcalde P, Garcia-Pouton N, Chumbita M. et al. Incidence of co-infections and superinfections in hospitalized patients with COVID-19: A retrospective cohort study. Clin Microbiol Infect. 2021; 27(1): 83-8.

https://doi.org/10.1016/j.cmi.2020.07.041

9. Zhou Q, Gao Y, Wang X, Liu R, Du P, Wang X, et al. Nosocomial infections among patients with COVID-19, SARS and MERS: A rapid review and meta-analysis. Ann Transl Med. 2020; 8(10): 1-14. https://doi.org/10.21037/atm-20-3324

10. Martos-Cabrera MB, Mota-Romero E, Martos-García R, Gómez-Urquiza JL, Suleiman-Martos N, Albendín-García L, et al. Hand hygiene teaching strategies among nursing staff: A systematic review. Int J Environ Res Public Health. 2019; 16(17): 1-13. https://doi.org/10.339o/ijerph16173039

11. Ambrosch A, Wahrburg K, Klawonn F. Bacterial load and pathogenic species on healthcare personnel attire: Implications of alcohol hand-rub use, profession, and time of duty. J Hosp Infect. 2019; 101(4): 414-21. https://doi.org/10.1016/j.jhin.2018.10.017

12. Reddy SC, Valderrama AL, Kuhar DT. Improving the use of personal protective equipment: Applying lessons learned. Clin Infect Dis. 2019; 69(Suppl 3): S165-S17O.

https://doi.org/10.1093/cid/ciz619

13. Ağalar C, Öztürk-Engin D. Protective measures for COVID-19 for healthcare providers and laboratory personnel. Turk J Med Sci. 2020; 50(SI-1): 578-84. https://doi.org/10.3906/sag-2004-132

14. Garritty C, Gartlehner G, Nussbaumer-Streit B, King VJ, Hamel C, Kamel C, et al. Cochrane rapid reviews methods group offers evidence-informed guidance to conduct rapid reviews. J Clin Epidemiol. 2021; 130: 13-22. https://doi.org/10.1016/j.jclinepi.2020.10.007

15. Liberati A, Altman DG, Tetzlaff J, Mulrow C, Gøtzsche PC, Ioannidis JPA, et al. The PRISMA statement for reporting systematic reviews and meta-analyses of studies that evaluate health care interventions: Explanation and elaboration. BMJ. 2009; 339: 1-27.

https://doi.org/10.1136/bmj.b270o

16. Lockwood C, Porritt K, Munn Z, Rittenmeyer L, Salmond S, Bjerrum M, et al. Chapter 2: Systematic reviews of qualitative evidence. In: Aromataris E, Munn Z (Eds.). JBI Manual for Evidence Synthesis. EE.UU.: JBI; 2020. https://doi.org/10.46658/JBIMES-20-03

17. Chan ST, Khong PCB, Wang W. Psychological responses, coping and supporting needs of healthcare professionals as second victims. Int Nurs Rev. 2016; 64(2): 242-62.

https://doi.org/10.1111/inr.12317

18. Bearman G, Bryant K, Leekha S, Mayer J, Munoz-Price LS, Murthy R, et al. Healthcare personnel attire in non-operating-room settings. Infect Control Hosp Epidemiol. 2014; 35(2): 107-21. https://doi.org/10.1086/675066

19. Munoz-Price LS, Arheart KL, Mills JP, Cleary T, Depascale D, Jimenez A, et al. Associations between bacterial contamination of health care workers' hands and contamination of white coats and scrubs. Am J Infect Control. 2012; 4O(9): e245-8. https://doi.org/10.1016/j.ajic.2012.03.032 
20. Goyal S, Khot SC, Ramachandran V, Shah KP, Musher DM. Bacterial contamination of medical providers' white coats and surgical scrubs: A systematic review. Am J Infect Control. 2019; 47(8): 994-1001. https://doi.org/10.1016/j.ajic.2019.01.012

21. Haun N, Hooper-Lane C, Safdar N. Healthcare personnel attire and devices as fomites: A systematic review. Infect Control Hosp Epidemiol. 2016; 37(11): 1367-73. https://doi.org/10.1017/ice.2016.192

22. Chen YC, Lin CF, Rehn YF, Chen JC, Chen PY, Chen $\mathrm{CH}$, et al. Reduced nosocomial infection rate in a neonatal intensive care unit during a 4-year surveillance period. J Chin Med Assoc. 2017; 80(7): 427-31. https://doi.org/10.1016/j.jcma.2017.02.006

23. Kampf G, Todt D, Pfaender S, Steinmann E. Persistence of coronaviruses on inanimate surfaces and their inactivation with biocidal agents. J Hosp Infect. 2020; 104(3): 246-51.

https://doi.org/10.1016/j.jhin.2020.01.022

24. Aboubakr HA, Sharafeldin TA, Goyal SM. Stability of SARS-CoV-2 and other coronaviruses in the environment and on common touch surfaces and the influence of climatic conditions: A review. Transbound Emerg Dis. 2021; 68(2): 296-312. https://doi.org/10.1111/tbed.13707

25. Wiener-Well Y, Galuty M, Rudensky B, Schlesinger Y, Attias D, Yinnon AM. Nursing and physician attire as possible source of nosocomial infections. Am J Infect Control. 2011; 39(7): 555-9.

https://doi.org/10.1016/j.ajic.2010.12.016

26. Liu S, Wang M, Wang G, Wu X, Guan W, Ren J. Microbial characteristics of nosocomial infections and their association with the utilization of hand hygiene products: A hospital-wide analysis of 78,344 cases. Surg Infect. 2017; 18(6): 676-83. https://doi.org/10.1089/sur.2017.037

27. Jordan-Garcia I, Esteban-Torné E, Bustinza-Arriortua A, de Carlos-Vicente JC, García-Soler P, Concha-Torre JA, et al. Trends in nosocomial infections and multidrug-resistant microorganisms in spanish pediatric intensive care units. Enferm. infecc. microbiol. clín. 2016; 34(5): 286-92.

https://doi.org/10.1016/j.eimc.2015.07.010

28. Giacobbe DR, Battaglini D, Ball L, Brunetti I, Bruzzone B, Codda G, et al. Bloodstream infections in critically ill patients with COVID-19. Eur J Clin Invest. 2020; 50(10): 1-8.

https://doi.org/10.1111/eci.13319

29. Burden M, Cervantes L, Weed D, Keniston A, Price CS, Albert RK. Newly cleaned physician unifor$\mathrm{ms}$ and infrequently washed white coats have similar rates of bacterial contamination after an 8-hour workday: A randomized controlled trial. J Hosp Med. 2011; 6(4): 177-82.

https://doi.org/10.1002/jhm.864

30. World Health Organization. Home care for patients with COVID-19 presenting with mild symptoms and management of their contacts. Geneva: WHO; 2020. https://bit.ly/3bHKoPo

31. Ajami NJ, Wong MC, Ross MC, Lloyd RE, Petrosino JF. Maximal viral information recovery from sequence data using VirMAP. Nat Commun. 2018; 9(1): 1-9.

https://doi.org/10.1038/s41467-018-05658-8

32. Rampelli S, Soverini M, Turroni S, Quercia S, Biagi E, Brigidi P, et al. ViromeScan: A new tool for metagenomic viral community profiling. BMC Genomics. 2016; 17: 1-9.

https://doi.org/10.1186/s12864-016-2446-3

33. Zapata-Giraldo J, Botero LE, Mejía ML, Escobar-Mora N, Ortiz-Trujillo I, Galeano BJ. Textiles funcionales como barrera de protección ante infecciones asociadas a la atención en salud. Revista EIA. 2018; 15(29): 13-29. https://doi.org/10.24050/reia.v15i29.1166 
34. Gomes-Rodrigues A, Romano-de Oliveira Gonçalves PJ, Ottoni CA, de Cássia-Ruiz R, Morgano MA, De Araújo WL, et al. Functional textiles impregnated with biogenic silver nanoparticles from Bionectria ochroleuca and its antimicrobial activity. Biomed Microdevices. 2019; 21(3): 56. https://doi.org/10.1007/s10544-019-0410-0

35. Liao C, Li Y, Tjong SC. Bactericidal and cytotoxic properties of silver nanoparticles. Int J Mol Sci. 2019; 20(2): 1-47. https://doi.org/10.339o/ijms20020449 